\title{
Specific Chromatographic Retentions on Polymer Pore Surface of Macroporous Spongy Monoliths
}

\author{
Tetsuya Tanigawa, ${ }^{1}$ Takuya Kubo, ${ }^{2}$ and Ken Hosoya*3 \\ ${ }^{1}$ Graduate School of Environmental Studies, Tohoku University, Aoba 6-6-20, Aramaki, Aoba-ku, Sendai, Miyagi 980-8579 \\ ${ }^{2}$ Graduate School of Engineering, Kyoto University, Katsura, Nishikyo-ku, Kyoto 615-8510 \\ ${ }^{3}$ Graduate School of Life and Environmental Sciences, Kyoto Prefectural University, \\ 1-5 Hangi-cho, Shimogamo, Sakyo-ku, Kyoto 606-8522
}

(Received May 14, 2012; CL-120584; E-mail: hosoya@kpu.ac.jp)

We examined chromatographic retention properties of macroporous spongy monolithic columns. Detailed chromatographic evaluations showed that planar compounds were strongly retained on poly(ethylene-co-vinyl acetate)-based monoliths, whereas sterically bulky or hydrophilic compounds were weakly retained. The comparison results with commonly used columns suggested that the specific retention abilities were a result of the differences in the polymer-chain orientation on polymer pore surface.

In the past few decades, monolithic columns containing silica or polymer-based materials have been widely developed in the field of liquid chromatographic separation. ${ }^{1-3}$ Previously, we reported that the macroporous poly(ethylene-co-vinyl acetate; EVA) spongy monolith having around $10 \mu \mathrm{m}$ pore size, effectively worked as a separation medium in reversed-phase chromatography. ${ }^{4}$ In particular, we showed the possibility for high-throughput separation based on a macroporous structure and the separation selectivity for polyaromatic hydrocarbons. ${ }^{5}$ In this letter, we describe the basic retention properties of the spongy monolith in point of polymer pore-surface structure. In reversed-phase chromatography, the retention properties can be examined as done in a previous study. ${ }^{6}$ In order to compare the retention properties of spongy monolith, columns were evaluated by liquid chromatography and as packed columns with octadecylsilylsilica particles (Sil-C18), ethylene glycol dimethacrylate (EDMA)-based particles, and other spongy monoliths having different contents of vinyl acetate units. ${ }^{11}$ In this letter, EVA containing $15 \%$ poly(vinyl acetate) against polyethylene is represented as EVA15. The spongy monolith prepared from linear low-density polyethylene is represented as PE.

Typical chromatograms of columns are shown in Figure 1. The comparison of EVA15 and EVA25 show that the content of vinyl acetate units played a role in determining the hydrophobic retention on spongy monolith. In addition, spongy monoliths provided better peak shape than packed column with EDMAbased particles. It was assumed that the peak tailing occurred because of the micropore structure ${ }^{7}$ on EDMA. On the other hand, there were no meso- and micropores in spongy monoliths in our previous study. Therefore, the spongy monolith gave a symmetric peak. Table 1 shows the separation coefficient of planar and sterically bulky solutes with nearly equal hydrophobicity. As shown in Table 1, separation coefficients for naphthalene and biphenyl have no significant differences in each column. In contrast, in the case of other solute combination, spongy monoliths showed selective retention for planar solutes and gave larger separation coefficient than those on EDMA and Sil-C18. Since polymer media has large steric selectivity for a

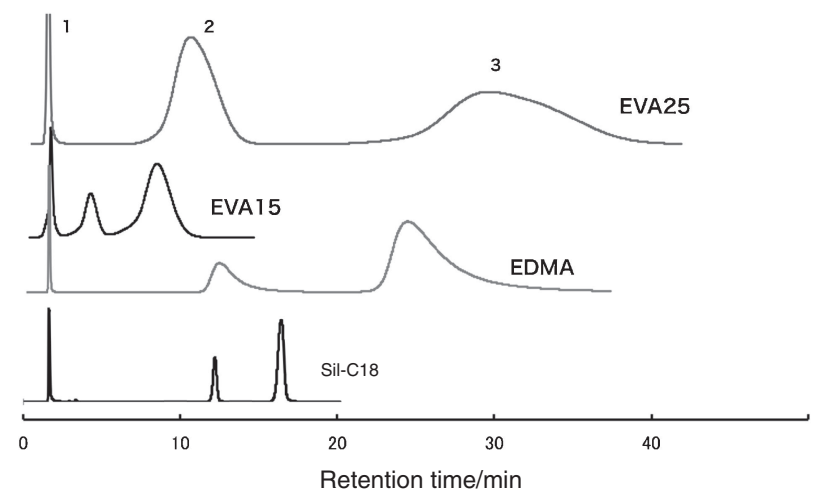

Figure 1. Typical chromatograms for test solutes. 1, Uracil; 2, o-terphenyl; 3, triphenylene. Mobile phase: methanol/water $=$ $80 / 20(\mathrm{v} / \mathrm{v})$, column size: $100 \times 4.6 \mathrm{~mm}$-i.d., Flow rate: 1.0 $\mathrm{mL} \min ^{-1}$, Detection: UV $254 \mathrm{~nm}$, Temperature: $40^{\circ} \mathrm{C}$.

Table 1. Comparison of separation coefficients between planar and nonplanar molecule ${ }^{\mathrm{a}}$

\begin{tabular}{|c|c|c|c|c|c|}
\hline Solute & EVA25 & EVA15 & $\mathrm{PE}$ & EDMA & Sil-C18 \\
\hline Naphtharene/biphenyl & 0.82 & 0.86 & 0.90 & 0.84 & 0.73 \\
\hline Fluorene/diphenylmethane & 1.56 & 1.71 & 1.67 & 1.17 & 1.08 \\
\hline Triphenylene/o-terphenyl & 2.46 & 2.76 & 1.97 & 1.53 & 1.17 \\
\hline
\end{tabular}

${ }^{a}$ Mobile phase: methanol/water $=70 / 30(\mathrm{v} / \mathrm{v})$. Separation coefficient is calculated by $\left(k_{\text {(planar molecule) }} / k_{\text {(nonplanar molecule) })}\right)$. Other conditions are described in Figure 1.

planar solute, the higher planar selectivity of spongy monoliths is noticeable. The results also showed that the selectivity for planar solutes was emphasized in larger molecule such as triphenylene.

Figure 2 that shows the comparison of the retention of terphenyl isomers and triphenylene gives a deeper insight into planar retentions. The elution order of terphenyl isomers were at ortho-, meta-, and para-positions on a spongy monolithic column. In addition, spongy monoliths strongly retained planar triphenylene compared to terphenyl isomers. The results indicated that the retention property of spongy monoliths was significantly different from those of particle-packed columns. Although para-isomer can be come to planar conformation, meta- and ortho-isomers are difficult to be taken planar conformation because of the steric hindrance of aromatic ring. Therefore, the results also supported the former consideration involving the planarity-selective retention on spongy monoliths.

As further examination of retention properties, we examined the polar selectivity of spongy monoliths by analyzing mono- 


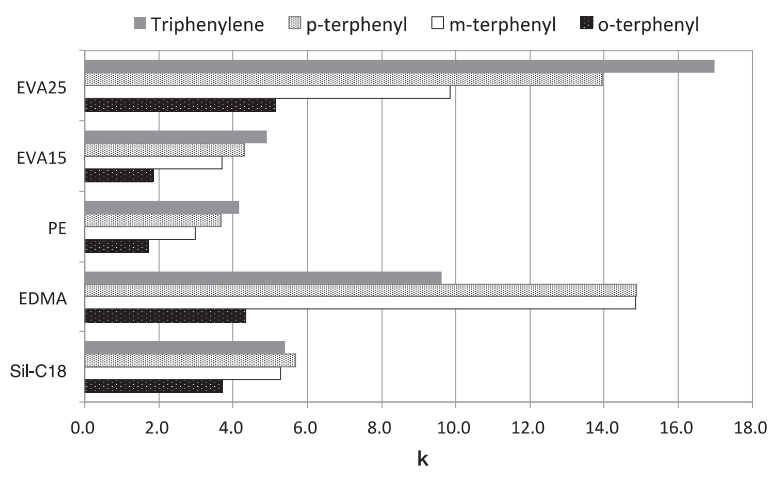

Figure 2. Comparison of retention for terphenyl isomers and triphenylene. Mobile phase: methanol/water $=85 / 15(\mathrm{v} / \mathrm{v})$. Other conditions are described in Figure 1.

substituted benzene compounds as solutes. In Figure 3, the retention rates for each solute toward benzene are plotted as radar charts. The hydrophobic parameter $\log P$ value of each solute was correlated with chromatographic retention parameter, $\log k$. Here, retention factor $k$ can be estimated as follows.

$$
k=\left(v_{(\text {retention volume of solute })}-v_{(\text {void volume })}\right) / v_{(\text {void volume })}
$$

In order to predict hydrophobic retention, the calculated $\log P$ of each solute against benzene is also shown as a dotted line in Figures $3 \mathrm{a}$ and $3 \mathrm{~b}$. Figure $3 \mathrm{~b}$ shows that the retention rate plot for Sil-C18 and EDMA closed relation with the predicted $\log P$. The results suggested that these columns retained these solutes mainly by hydrophobic interaction. On the other hand, spongy monoliths provided comparable retention to EDMA for hydrophobic solutes like toluene and anisole. However, smaller retention was observed for hydrophilic solutes such as phenol, benzoic acid, and benzamide than the predicted $\log P$ value in Figure 3a. Since typical polymer-based stationary phases have greater retention toward hydrophilic solutes, the lower selectivity for hydrophilic compounds implies specific retention property in spongy monoliths. We assumed that the specific retention abilities with higher selectivity for planar solutes and lower retention for hydrophilic solutes on spongy monoliths were because of the differences in the polymer-chain orientation on polymer pore surface. Our thermal analysis result supported that polymer-chain orientation on EVA decreases with respect to vinyl acetate content. ${ }^{8}$ The results suggested that polymer-chain orientation is stronger in pure polyethylene. In other words, vinyl acetate units are roughly oriented compared to polyethylene. Therefore, the flexible brush-like alkyl chain network like Sil-C18 can be generated on EVA surface. In addition, the carbonyl-group stationary phase retains polyaromatic hydrocarbons by specific multiple carbonyl- $\pi$ interactions. ${ }^{9,10}$ Thus, large planar solutes can be selectively retained. Also, it was assumed that hydrated hydrophilic functional groups on a polar solute blocked the planar retention of benzene ring on the surface of spongy monoliths. In brief, we concluded that the specific planar recognition was dominant on spongy monoliths, and further studies are under progress.

This study was supported by the Ministry of the Environment, Government of Japan, through the Environment Research and Technology Development Fund (No. B-0806).
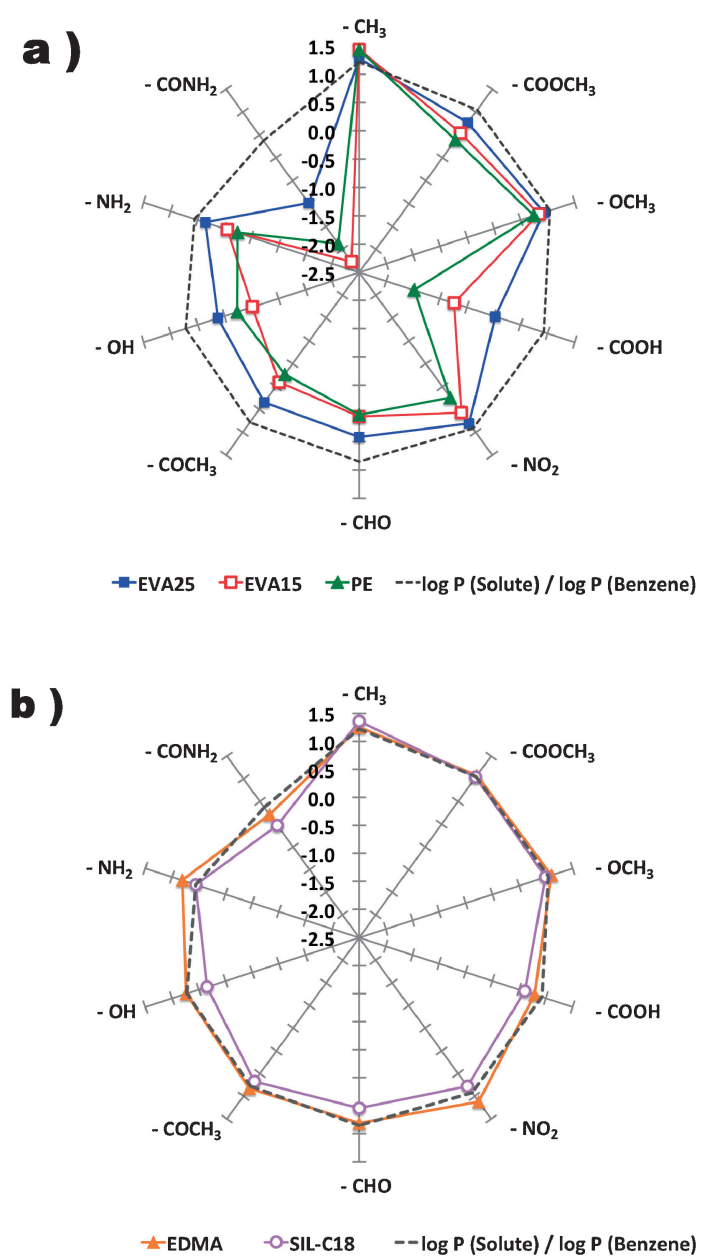

Figure 3. Retention rates for each solute toward benzene on each column $\left(k_{\text {(solute) }} / k_{\text {(benzene) }}\right)$. Spongy monolithic columns: a) Sil-C18 and EDMA columns; b) mobile phase: methanol/ water $/$ formic acid $=40 / 60 / 0.1(\mathrm{v} / \mathrm{v} / \mathrm{v})$. Other conditions are described in Figure 1.

Paper based on a presentation made at the International Association of Colloid and Interface Scientists, Conference (IACIS2012), Sendai, Japan, May 13-18, 2012.

\section{References and Notes}

1 N. Tanaka, H. Kobayashi, K. Nakanishi, H. Minakuchi, N. Ishizuka, Anal. Chem. 2001, 73, 420A.

2 F. Svec, J. Sep. Sci. 2005, $28,729$.

3 K. Hosoya, N. Hira, K. Yamamoto, M. Nishimura, N. Tanaka, Anal. Chem. 2006, 78, 5729.

4 T. Kubo, F. Watanabe, K. Kaya, K. Hosoya, Chem. Lett. 2008, 37, 950.

5 T. Tanigawa, K. Kato, Y. Watabe, T. Kubo, K. Hosoya, J. Sep. Sci. 2011, 34, 2193.

6 K. Kimata, K. Iwaguchi, S. Onishi, K. Jinno, R. Eksteen, K. Hosoya, M. Araki, N. Tanaka, J. Chromatogr. Sci. 1989, 27, 721.

7 K. Hosoya, M. Teramachi, N. Tanaka, A. Kobayashi, T. Kanda, Y. Ohtsu, Anal. Chem. 2001, 73, 5852.

8 X. M. Shi, J. Zhang, J. Jin, S. J. Chen, eXPRESS Polym. Lett. 2008, 2, 623.

9 M. M. Rahman, M. Takafuji, H. R. Ansarian, H. Ihara, Anal. Chem. 2005, 77, 6671.

10 A. A. Rana, M. Takafuji, H. Ihara, J. Chromatogr., A 2009, 1216, 7440.

11 Supporting Information is available electronically on the CSJ-Journal Web site, http://www.csj.jp/journals/chem-lett/index.html. 